\title{
ON THE QUATERNIONIC SECTIONAL CURVATURE OF AN INDEFINITE QUATERNIONIC K̈̈HLER MANIFOLD
}

\author{
By \\ Eduardo GARCíA-Rio* and M. Elena VÁZqueZ-ABAL*
}

\section{Introduction}

The quaternionic sectional curvature of an indefinite quaternionic Kähler manifold is investigated in [6], where it is shown that its treatment presents certain analogies with that of the holomorphic sectional curvature of an indefinite Kähler manifold [1].

An important feature of indefinite metrics is the existence of null geodesics, and the study of the Jacobi operator along such geodesics. A simple examination of the curvature tensor of an indefinite Kähler manifold of constant holomorphic sectional curvature shows that its restriction to degenerate holomorphic planes vanishes identically. Such condition $R(U, J U, J U, U)=0$ is shown in [3] to be strictly weaker than constant holomorphic sectional curvature. In fact, the product $M_{1}(c) \times M_{2}(c)$ of two positive definite Kähler manifolds endowed with the metric $g=g_{1} \oplus\left(-g_{2}\right)$ satisfies $R(U, J U, J U, U)=0$ but its holomorphic sectional curvature is not constant, unless $c=0$.

When one considers an indefinite quaternionic Kähler manifold of constant $q$-sectional curvature, the curvature tensor is expressed in terms of the metric and the almost complex structures of the quaternionic structure. From that expression it immediately follows that

$$
R(U, \phi U, \phi U, U)=0, \quad \phi=I, J, K,
$$

where $\{I, J, K\}$ is any local basis of the bundle of almost complex structures on $M$.

The aim of this paper is to investigate such condition (1), and to prove that it is characteristic of indefinite quaternionic space forms. This makes a significant difference in the study of the curvature of indefinite quaternionic Kähler manifolds with respect to the complex case. We will show the following.

* Partially supported by a Project DGICYT, PB89-0571-C02-01 (Spain).

Received August 9, 1993. Revised February 15, 1994. 
THEOREM A. Let $(M, g, V)$ be an indefinite quaternionic Kähler manifold. Then $M$ is an indefinite quaternionic space form if and only if

$$
R(U, \phi U, \phi U, U)=0
$$

for all null vector $U, \phi=I, J, K$.

Theorem $\mathrm{A}$ has been stated previously in [6, Th. 7.3], but the proof there seemed to be inadequate because of the lack of convergence, in general, of a certain real sequence. Moreover, perhaps that approach is too difficult to repair, because of a counterexample [3] to the complex case [1, Th. 6.5]. For this reason, we provide a completely different proof.

Finally, considering the expression of the curvature tensor of an indefinite quaterionic space form, we obtain the form of the Jacobi operator along null geodesics for such spaces. As a direct application, we show the nonexistence of conjugate points along null geodesics in indefinite quaternionic space forms.

We will follow the notations of [6] and we refer to it (see also [2], [7]), for the definitions of the $q$-sectional curvature and some basic facts concerning the curvature tensor of an indefinite quaternionic Kähler manifold. All manifolds are assumed to be connected.

\section{Preliminaries}

Let $(M, g, V)$ be an indefinite almost quaternionic manifold, i.e., $V$ a 3dimensional bundle of almost complex structures on $M$, and $g$ an indefinite metric of signature $(4 p, 4 q),(p+q=n)$ satisfying

$$
g(\phi X, \phi Y)=g(X, Y)
$$

for all vector fields $X, Y \in \mathscr{X}(M), \phi=I, J, K,\{I, J, K\}$ being a local basis of $V$.

$(M, g, V)$ is said to be indefinite Kähler if the bundle $V$ is parallel with respect to the metric connection $\nabla$ induced by $g$. As a consequence of such condition, one has the following identities for the curvature tensor [6]

$$
\begin{aligned}
R(X, Y, Z, W)= & R(X, Y, I Z, I W) \\
& -\frac{1}{n+2}\{g(Z, J W) \operatorname{Ric}(X, J Y)+g(Z, K W) \operatorname{Ric}(X, K Y)\}, \\
R(X, Y, Z, W)= & R(X, Y, J Z, J W) \\
& -\frac{1}{n+2}\{g(Z, I W) \operatorname{Ric}(X, I Y)+g(Z, K W) \operatorname{Ric}(X, K Y)\},
\end{aligned}
$$




$$
\begin{aligned}
R(X, Y, Z, W)= & R(X, Y, K Z, K W) \\
& -\frac{1}{n+2}\{g(Z, I W) \operatorname{Ric}(X, I Y)+g(Z, J W) \operatorname{Ric}(X, J Y)\},
\end{aligned}
$$

for all $X, Y, Z, W$ vector fields on $M$, where $R \imath c$ is the Ricci tensor of $M$, $\operatorname{dim} M=4 n$.

Throughout this paper we assume the existence of null vectors $U,(g(U, U)$ $=0$ ) and hence it must be $p, q \geqq 1$. An important consequence of this fact is that the metric is Einstein, because $\operatorname{dim} M=4 n \geqq 8$.

LEMMA 1. Let $(M, g, V)$ be an indefinite almost quaternionic manifold, and $U$ a null vector, $U \in T_{m} M$. Then there exist orthogonal vectors $X, Y \in T_{m} M$ spanning a totally real plane such that $U=X+Y$.

Proof. Let $\left\{E_{1}, \cdots, E_{4 n}\right\}$ be an orthonormal basis at $m$, and write $U=$ $\sum_{i=1}^{4 p} \lambda^{i} E_{i}+\sum_{j=4 p+1}^{4 n} \mu^{j} E_{j}$, where the first $4 p$-vectors in the basis above are assumed to be spacelike, and the last $4(n-p)$ timelike. Put $X^{\prime}=\sum_{i=1}^{4} \lambda^{i} E_{i}, Y^{\prime}=$ $\sum_{j=4 p+1}^{4 n} \mu^{j} E_{j}$, and consider the subspace $W$ spanned by $\left\{X^{\prime}, I X^{\prime}, J X^{\prime}, K X^{\prime}, Y^{\prime}\right.$, $\left.I Y^{\prime}, J Y^{\prime}, K Y^{\prime}\right\}$.

Let $X_{1}$ be an arbitrary spacelike vector in $W$, and consider the 4-plane $W_{1}=$ $Q\left(X_{1}\right)$, spanned by $\left\{X_{1}, I X_{1}, J X_{1}, K X_{1}\right\}$. Let $W_{2}$ denote the complementary orthogonal subspace of $W_{1}$ in $W$. Since $W_{1}$ is invariant by the quaternionic structure $\phi=I, J, K$ and $W_{2}$ is orthogonal to $W_{1}, W_{2}$ is also invariant.

Now, since $U \in W$, there exist $\alpha \in W_{1}$ and $\beta \in W_{2}$ such that $U=\alpha+\beta$. The result follows if we put $X=\alpha, Y=\beta$.

In order to analize (1), the following identities will be used extensively,

LEMMA 2. Let $(M, g, V)$ be an inde finte quaternionic Kähler manifold satisfying condition (1). Then for each pair of orthogonal vectors $X, Y \in \mathfrak{X}(M)$ with $g(X, X)=-g(Y, Y)$,

(a) $R(X, \phi X, X, \phi Y)-R(X, \phi X, \phi X, Y)$

$$
=R(Y, \phi Y, \phi Y, X)-R(Y, \phi Y, Y, \phi X)
$$

(b) $R(X, \phi X, \phi X, X)+R(Y, \phi Y, \phi Y, Y)=2 R(X, \phi X, Y, \phi Y)$

$$
+2 R(X, \phi Y, Y, \phi X)+R(X, \phi Y, X, \phi Y)+R(Y, \phi X, Y, \phi X)
$$

for all $\phi=I, J, K$.

Proof. Since $X$ is orthogonal to $Y$, and $g(X, X)=-g(Y, Y)$, then $X+Y$ 
and $X-Y$ are null vectors, and hence

$$
R(X \pm Y, \phi X \pm \phi Y, \phi X \pm \phi Y, X \pm Y)=0 .
$$

Now the result follows by linearizing this expression.

The next proposition shows the significance of condition (1) in terms of the Jacobi operator along null geodesics

Proposition 3. An indefinite quaternionic Kähler manifold, $(M, g, V)$, satisfies condition (1) if and only if

$$
R(U, \phi U) \phi U=c_{U}^{\phi} U
$$

for all null vectors $U$, and some functions $c_{U}^{\phi}, \phi=I, J, K$.

Proof. It is clear that (3) implies (1). Conversely, if (1) holds, then $R(U, \phi U) \phi U \in\langle U\rangle^{\perp}$. Since $U \in\langle U\rangle^{\perp}$, it will be enough to show that $g(R(U, \phi U) \phi U, V)=0$, for all null vectors $V \in\langle U\rangle^{\perp}$.

For each null $V \in\langle U\rangle^{\perp}, U+s V$ is a null vector, and hence

$$
R(U+s V, \phi U+s \phi V, \phi U+s \phi V, U+s V)=0 \text {. }
$$

Linearizing this expression, and considering the coefficient of $s$, we have

$$
0=2 R(U, \phi U, \phi U, V)+2 R(U, \phi U, \phi V, U)=4 g(R(U, \phi U) \phi U, V),
$$

which shows that $R(U, \phi U) \phi U=c_{\&}^{\phi} U$ for every null vector $U, \phi=I, J, K$.

LemMA 4. Let $(M, g, V)$ be a 4n-dimensional indefinite quaternionic Kähler manifold satisfying condition (1), and $U \in T_{m} M$ a null vector. If $U=X+Y$ for some $X, Y$ spanning a totally real plane as in Lemma 1 , then

$$
c_{U}^{I}=4 \varepsilon_{Z} R(U, Z, Z, U)-\frac{3 S c}{4 n(n+2)} \varepsilon_{Z}\left\{g(U, J Z)^{2}+g(U, K Z)^{2}\right\}
$$

for all unit $Z$ such that $\langle\{X, I X\}\rangle \perp Z \perp\langle\{Y, I Y\}\rangle$

$$
c_{U}^{J}=4 \varepsilon_{Z} R(U, Z, Z, U)-\frac{3 S c}{4 n(n+2)} \varepsilon_{Z}\left\{g(U, I Z)^{2}+g(U, K Z)^{2}\right\}
$$

for all unit $Z$ such that $\langle\{X, J X\}\rangle \perp Z \perp\langle\{Y, J Y\}\rangle$

$$
c_{U}^{K}=4 \varepsilon_{Z} R(U, Z, Z, U)-\frac{3 S c}{4 n(n+2)} \varepsilon_{Z}\left\{g(U, I Z)^{2}+g(U, J Z)^{2}\right\}
$$

for all unit $Z$ such that $\langle\{X, K X\}\rangle \perp Z \perp\langle\{Y, K Y\}\rangle$, where Sc denotes the scalar curvature of $M$.

Proof. Let us show (4). Let $X, Y$ be vectors as in Lemma 1 such that 
$U=X+Y$, and consider $V$ a null vector such that $g(U, V)=-1 / 2,\left(V=1 / 4 g(X, X)^{-1}\right.$ $(Y-X))$. Let $Z$ be an arbitrary unit vector such that $Z \in\langle\{X, I X\}\rangle^{\perp}$ and $Z \in$ $\langle\{Y, I Y\}\rangle^{\perp}$. Let $\omega_{t}$ denote the vector $\omega_{t}=1 / \sqrt{t}\left(U+t \varepsilon_{Z} V\right)$, where $\varepsilon_{Z}=g(Z, Z)$.

Since $\langle\{X, I X\}\rangle \perp Z \perp\langle\{Y, I Y\}\rangle$, it is clear that $Z \in\langle\{U, I U\}\rangle^{\perp}, Z \in$ $\langle\{V, I V\}\rangle^{\perp}$, and moreover that

$$
\langle\{Z, I Z\}\rangle \perp\left\langle\left\{\omega_{t}, I \omega_{t}\right\}\right\rangle, \quad g(Z, Z)=-g\left(\omega_{t}, \omega_{t}\right) .
$$

Hence, condition (b) in Lemma 2 gives us,

$$
\begin{aligned}
R(Z, I Z, I Z, Z)+R\left(\omega_{t}, I \omega_{t}, I \omega_{t}, \omega_{t}\right)=2 R\left(Z, I Z, \omega_{t}, I \omega_{t}\right) \\
\quad+2 R\left(Z, I \omega_{t}, \omega_{t}, I Z\right)+R\left(Z, I \omega_{t}, Z, I \omega_{t}\right)+R\left(\omega_{t}, I Z, \omega_{t}, I Z\right),
\end{aligned}
$$

and thus

$$
\begin{aligned}
& t R(Z, I Z, I Z, Z)+\frac{1}{t} R\left(U+t \varepsilon_{Z} V, I\left(U+t \varepsilon_{Z} V\right), I\left(U+t \varepsilon_{Z} V\right), U+t \varepsilon_{Z} V\right) \\
& =2 R\left(Z, I Z, U+t \varepsilon_{Z} V, I\left(U+t \varepsilon_{Z} V\right)\right)+2 R\left(Z, I\left(U+t \varepsilon_{z} V\right), U+t \varepsilon_{Z} V, I Z\right) \\
& \quad+R\left(Z, I\left(U+t \varepsilon_{Z} V\right), Z, I\left(U+t \varepsilon_{Z} V\right)\right)+R\left(U+t \varepsilon_{Z} V, I Z, U+t \varepsilon_{Z} V, I Z\right) .
\end{aligned}
$$

Linearizing previous expression and taking limits as $t \rightarrow 0$, one gets

$$
\begin{aligned}
& 2 \varepsilon_{Z} R(U, I U, I U, V)+2 \varepsilon_{Z} R(U, I U, I V, U) \\
& \quad=2 R(U, I U, Z, I Z)+2 R(U, I Z, Z, I U)+R(U, I Z, U, I Z)+R(Z, I U, Z, I U) .
\end{aligned}
$$

Using the identities (2), it follows that $R(U, I U, I V, U)=R(U, I U, I U, V)$, and hence

$$
\begin{aligned}
-2 \varepsilon_{Z} c_{U}^{I}= & 2 R(U, I U, Z, I Z)+2 R(U, I Z, Z, I U) \\
& +R(U, I Z, U, I Z)+R(Z, I U, Z, I U) .
\end{aligned}
$$

Once again, from (2), one has $R(Z, I U, Z, I U)=R(U, I Z, U, I Z)$, and hence (7) becomes

$$
-\varepsilon_{Z} c_{U}^{I}=R(U, I U, Z, I Z)+R(U, I Z, Z, I U)+R(U, I Z, U, I Z) .
$$

Once again, from (2), and using the first Bianchi identity, one gets after some calculations

$$
\begin{aligned}
c_{U}^{I}= & \varepsilon_{Z} R(U, Z, Z, U)+3 \varepsilon_{Z} R(U, I Z, I Z, U) \\
& -\frac{3 S c}{4 n(n+2)} \varepsilon_{Z}\left\{g(Z, J U)^{2}+g(Z, K U)^{2}\right\} .
\end{aligned}
$$

If we put $Z=I Z$ in (8), one gets $R(U, Z, Z, U)=R(U, I Z, I Z, U)$, and the result is obtained from previous equation. The remaining identities (5) and (6) are obtained in the same way. 
COROLLARY 5. Let $(M, g, V)$ be an indefinite quaternionic Kähler manifold satisfying condition (1). Then

for each null $U$.

$$
c_{U}^{I}=c_{U}^{J}=c_{U}^{K}
$$

Proof. Let $U$ be an arbitrary null vector, $U \in T_{m} M$, and show that $c_{U}^{I}=c_{U}^{X}$. Put $U=X+Y$ for some orthogonal $X, Y$ spanning a totally real plane as in Lemma 1, and take $Z=J X$. Since $\langle\{X, I X\}\rangle \perp J X \perp\langle\{Y, I Y\}\rangle$ and $\langle\{X, K X\}\rangle$ $\perp J X \perp\langle\{Y, K Y\}\rangle$, it follows from (4) and (6) in previous lemma that $c_{U}^{I}=c_{U}^{K}$. The remaining cases are obtained in the same way.

COROLlaRY 6. Let $(M, g, V)$ be an indefinite quaternionic Kähler manifold of $\operatorname{dim} M>8$ satisfying condition (1), and $U \in T_{m} M$ a null vector. If $U=X+Y$ for some orthogonal $X, Y$ spanning a totally real plane as in Lemma 1 , then

$$
c_{U}^{\phi}=4 \varepsilon_{Z} R(U, Z, Z, U), \quad \phi=I, J, K,
$$

for all unit $Z$ such that $Q(X) \perp Z \perp Q(Y)$.

PROoF. It follows directly from (4), (5) and (6) in previous lemma.

\section{Constancy of the quaternionic sectional curvature}

In [6], the following criteria for the constancy of the quaternionic sectional curvature is obtained.

LEMMA 7. Let $(M, g, V)$ be an indefinite quaternionic Kähler manifold of real dimension $4 n \geqq 8$. If for any $m \in M$, there exists a local basis $\{I, J, K\}$ of $V$ such that $R(X, \phi X, \phi X, Y)=0$, for every orthonormal vectors $X, Y \in T_{m} M$ spanning a totally real plane and some $\phi=I, J, K$, then $M$ is an indefinite quaternionic space form.

Next theorem gives conditions on the Jacobi operator along spacelike, timelike and null geodesics, each of them equivalent to the constancy of the $q$-sectional curvature.

THEOREM 8. For an indefinite quaternionic Kähler manifold, $(M, g, V)$, the following conditions are equivalent

(a) The q-sectional curvature is constant,

(b) $R(X, \phi X) \phi X \sim X, \quad$ for all spacelike vectors $X$. 
(c) $R(X, \phi X) \phi X \sim X$, for all timelike vectors $X$.

(d) $R(U, \phi U) \phi U=0$, for all null vectors $U$.

where $\sim$ means proportional, and $\phi=I, J, K$.

Proof. If the $q$-sectional curvature is a constant $c$, then $R(X, \phi X) \phi X=$ $\operatorname{cg}(X, X) X$, which shows the necessity of (b). To see the sufficiency, we will look at $R(X, \phi X, \phi X, Y)$ for all possible $\{X, Y\}$ spanning a totally real plane, and use Lemma 7. If $X$ is spacelike, then by (b), $R(X, \phi X, \phi X, Y)=0$.

If $X$ is timelike and $Y$ is also timelike then there exists an unit spacelike vector $Z$ such that $Q(X) \perp Z \perp Q(Y)$; hence $R(\lambda Z+X, \lambda \phi Z+\phi X, \lambda \phi Z+\phi X, Y)$ $=0$, for $|\lambda|>1$, and we get $R(X, \phi X, \phi X, Y)=0$. If $Y$ is spacelike, then $\lambda Y+\mu X$ satisfies $g(\lambda Y+\mu X, \lambda Y+\mu X)=\lambda^{2}-\mu^{2}$ and $Q(\lambda Y+\mu X) \perp Q(\lambda X+\mu Y)$ hence $R(\lambda Y+\mu X, \lambda \phi Y+\mu \phi X, \lambda \phi Y+\mu \phi X, \lambda X+\mu Y)=0$, for $\lambda^{2}-\mu^{2}>0$. Linearizing this expression it follows that $R(X, \phi X, \phi X, Y)=0$.

The equivalence between (c) and (a) is obtained in an analogous way.

To prove that (d) is equivalent to (a), we proceed as follows. If the $q$ sectional curvature is a constant $c$, for every tangent vector $X, R(X, \phi X) \phi X=$ $\operatorname{cg}(X, X) X$, and hence $R(U, \phi U) \phi U=0$ for every null vector $U$.

For the sufficiency we use again Lemma 7. Let $X$ and $Y$ be unitary tangent vectors spanning a totally real plane. If $g(X, X)=-g(Y, Y)$, then $X+Y$ and $X-Y$ are null vectors, and therefore using (d)

$$
R(X+Y, \phi X+\phi Y, \phi X+\phi Y, X-Y)-R(X-Y, \phi X-\phi Y, \phi X-\phi Y, X+Y)=0,
$$

from which we get

$$
R(X, \phi X, \phi X, Y)-R(Y, \phi Y, \phi Y, X)=0 .
$$

Considering now (10) and (a) in Lemma 2, it follows that $R(X, \phi X, \phi X, Y)=0$.

Now, if $g(X, X)=g(Y, Y)$ then consider $Z$ a unit vector with $g(Z, Z)=$ $-g(X, X)$ such that $Q(X) \perp Q(Z) \perp Q(Y)$. Since $\{X, \lambda Z+Y\}$ spanns a totally real plane, it follows that $R(X, \phi X, \phi X, \lambda Z+Y)=0$ for values of $|\lambda|>1$, and hence $R(X, \phi X, \phi X, Y)=0$.

It is clear now that what remains for proving that condition (1) is equivalent to constant $q$-sectional curvature in quaternionic Kähler manifolds is to show that the functions $c_{U}^{\phi}$ vanish identically.

PROPOSITION 9. Let $\left(M^{4 n}, g, V\right)$ be an indefinite quaternionic Kähler manifold satisfying condition (1). Then the Ricci tensor satisfies 


$$
\operatorname{Ric}(U, U)=(n+4) c_{G}^{\phi},
$$

for any null vector $U \in T_{m} M$.

Proof. Let $m$ be an arbitrary point on $M, U$ a null vector $U \in T_{m} M$, and consider $X, Y \in T_{m} M$ unit vectors spanning a totally real plane with $U=k(X+Y)$ as in Lemma 1. Decompose the tangent space $T_{m} M=\langle X, I X, J X, K X\rangle \oplus$ $\langle Y, I Y, J Y, K Y\rangle \oplus\left\langle Z_{1}, \cdots, Z_{4 n-8}\right\rangle$, where $\left\langle Z_{1}, \cdots, Z_{4 n-8}\right\rangle$ is an orthonormal basis of $(Q(X) \oplus Q(Y))^{\perp}$. With respect to such a basis, the Ricci tensor has the expression

$$
\begin{aligned}
\operatorname{Ric}(U, U)= & R(X, U, U, X)-R(Y, U, U, Y)+R(I X, U, U, I X) \\
& -R(I Y, U, U, I Y)+R(J X, U, U, J X)-R(J Y, U, U, J Y) \\
& +R(K X, U, U, K X)-R(K Y, U, U, K Y) \\
& +\sum_{i=1}^{4 n-8} g\left(Z_{i}, Z_{i}\right) R\left(Z_{i}, U, U, Z_{i}\right) .
\end{aligned}
$$

Now,

$$
\begin{aligned}
& R(X, U, U, X)-R(Y, U, U, Y) \\
& \quad=k^{2} R(X, X+Y, X+Y, X)-k^{2} R(Y, X+Y, X+Y, Y)=0,
\end{aligned}
$$

and

$$
\begin{aligned}
& R(\phi X, U, U, \phi X)-R(\phi Y, U, U, \phi Y) \\
& \quad=k^{2} R(\phi X, X+Y, X+Y, \phi X)-k^{2} R(\phi Y, X+Y, X+Y, \phi Y) \\
& \quad=k^{2} R(X+Y, \phi(X+Y), \phi(X+Y),(X-Y)) \\
& \quad=g\left(c_{U}^{\delta} U, k^{-1}(X-Y)\right)=2 c_{U}^{\phi} .
\end{aligned}
$$

Using the identities in Lemma 4 and Corollaries 5 and 6, we obtain the result from (12).

Now, we can prove the anounced

THEOREM A. Let $(M, g, V)$ be an indefinite quaternionic Kähler manifold. Then $M$ is an indefinite quaternionic space form if and only if

$$
R(U, \phi U, \phi U, U)=0
$$

for all null vectors $U, \phi=1, J, K$.

PROOF. Since any indefinite quaternionic Kähler manifold of $\operatorname{dim} M=4 n \geqq 8$ is Einstein, it follows from (11) that 


$$
(n+4) c_{U}^{b}=R i c(U, U)=0 .
$$

This shows that $c^{\phi}=0$ for all null $U, \phi=I, J, K$, and the result follows from (d) in Theorem 8.

As a direct consequence of Proposition 9 and Theorem $A$, we state the following result on the boundedness of the $q$-sectional curvature.

THEOREM 10. Let $(M, g, V)$ be an indefinite quaternionic Kähler manifold. If the q-sectional curvature is bounded from above (or from below) on spacelike quaternionic planes and bounded from below (or from above) on timelike quaternonic planes, then it is constant.

Proof. Let $U \in T_{m} M$ be an arbitrary null vector, and $Z$ an unit vector orthogonal to $U$. Then $Z_{n}=U+1 / n Z$ is a sequence of non null vectors aproximating $U$. The $Z_{n}$ are spacelike or timelike if and only if $Z$ is so, and hence

$$
R\left(Z_{n}, \phi Z_{n}, \phi Z_{n}, Z_{n}\right) \leqq A g\left(Z_{n}, Z_{n}\right)^{2}
$$

for some constant $A$ if $g(Z, Z)=1$, and

$$
R\left(Z_{n}, \phi Z_{n}, \phi Z_{n}, Z_{n}\right) \geqq B g\left(Z_{n}, Z_{n}\right)^{2},
$$

for some real $B$, if $g(Z, Z)=-1$.

Taking limits in previous expressions as $n \rightarrow \infty$, one gets

$$
R(U, \phi U, \phi U, U) \leqq 0, \quad R(U, \phi U, \phi U, U) \geqq 0,
$$

and hence $R(U, \phi U, \phi U, U)=0$ for all null $U$. Now the result follows from Theorem A.

REMARK 11. Using the expression obtained in [6] for the curvature tensor of an indefinite quaternionic space form,

$$
\begin{aligned}
R(X, Y) Z= & \frac{c}{4}\{g(Y, Z) X-g(X, Z) Y+g(I Y, Z) I X-g(I X, Z) I Y \\
& +g(J Y, Z) J X-g(J X, Z) J Y+g(K Y, Z) K X-g(K X, Z) K Y \\
& +2 g(X, I Y) I Z+2 g(X, J Y) J Z+2 g(X, K Y) K Z\}
\end{aligned}
$$

it follows that for any totally real degenerate plane $\pi=\langle\{X, Y\}\rangle$,

$$
R(X, Y, Y, X)=0
$$

and

$$
R(X, \phi X, \phi Y, Y)=0, \quad \phi=I, J, K
$$


Now, if $U$ is a null vector, then $\{U, \phi U\}$ spanns a degenerate totally real plane $(\phi=I, J, K)$, and hence, applying either (14) or (15) to $\{U, \phi U\}$, it must be $R(U, \phi U, \phi U, U)=0$, which shows that the quaternionic sectional curvature is constant, according to previous Theorem A. Hence, it is clear that for an indefinite quaternionic Kähler manifold, conditions (1), (14) and (15) are, each of them, equivalent to constant quaternionic sectional curvature. (Compare with the results in [3] for the complex case).

\section{The Jacobi equation along null geodesics}

Finally, we shall solve the Jacobi equation along a null geodesic $\gamma$ in an indefinite quaternionic space form. We will follow the notations in [4]. Let $\bar{\gamma}^{\perp}$ denote the nondegenerate normal bundle of $\gamma, \bar{\gamma}^{\perp}=\gamma^{\perp} /\left\langle\gamma^{\prime}\right\rangle, \bar{R}_{\gamma}$ the projection on $\bar{\gamma}^{\perp}$ of the Jacobi operator, $R_{\gamma} X=R\left(X, \gamma^{\prime}\right) \gamma^{\prime}$ and $\bar{X}=\pi(X)$ for any vector field $X$ along $\gamma$, with $\pi: \gamma^{\perp} \mapsto \bar{\gamma}^{\perp}$ the projection. Also let $\bar{X}^{\prime}$ be the covariant derivative of $\bar{X}$ along $\gamma$ defined by $\bar{X}^{\prime}=\pi\left(\nabla_{\gamma}, X\right)$, for any $X$ vector field along $\gamma$.

Since $\gamma^{\prime}(t) \in T_{\gamma(t)} M$ is a null vector, there exist spacelike and timelike $X, Y$ $\in T_{\gamma(t)} M$ spanning a totally real plane such that $\gamma^{\prime}(t)=X+Y$. Complete $Q(X) \oplus$ $Q(Y)$ to an orthogonal basis of $T_{\gamma(t)} M$, and consider the parallel frame along $\gamma$ given by

$$
\left\{X, Y, I_{t} X, I_{t} Y, J_{t} X, J_{t} Y, K_{t} X, K_{t} Y, Z_{1}, \cdots, Z_{4 n-8}\right\},
$$

where $\left\{I_{t}, J_{t}, K_{t}\right\}$ denotes a rotating basis of the bundle $V$ along $\gamma(t)$ such that the covariant derivatives $\nabla_{\gamma^{\prime}(t)} \phi_{t}$ vanish for $\phi=I, J, K$. Hence the projection

$$
\left\{\overline{I_{t} X}, \overline{I_{t} Y}, \overline{J_{t} X}, \overline{J_{t} Y}, \overline{K_{t} X}, \overline{K_{t} Y}, \bar{Z}_{1} \cdots, \bar{Z}_{4 n-8}\right\},
$$

of the basis above, is a parallel basis of $\bar{\gamma}^{\perp}$. Considering the expression (13) of the curvature tensor of an indefinite quaternionic Kähler manifold of constant quaternionic sectional curvature $c$, the matrix of the Jacobi operator $\bar{R}_{r}$ with respect to the basis above is block diagonal

$$
\bar{R}_{r}=\left(\begin{array}{cccc}
L & & & \\
& L & & \\
& & L & \\
& & & 0
\end{array}\right)
$$

where $L$ is the $2 \times 2$ matrix

$$
L=\left(\begin{array}{ll}
\frac{3 c}{4} g(X, X) & -\frac{3 c}{4} g(X, X) \\
\frac{3 c}{4} g(X, X) & -\frac{3 c}{4} g(X, X)
\end{array}\right)
$$


Now, we state the following result on the existence of conjugate points along null geodesics.

THEOREM 12. Let $(M, g, V)$ be an indefinite quaternionic space form. Then there are no conjugate points along null geodesics.

Proof. Let $\bar{A}(t)$ be a solution of the Jacobi equation, $\bar{A}^{\prime \prime}+\bar{R}_{\gamma} \circ \bar{A}=0$, satisfying the initial conditions $\bar{A}(0)=0, \bar{A}^{\prime}(0)=\bar{I} \bar{d}$. Then, $\gamma$ has a conjugate point of zero at $t$ if and only if $\operatorname{det} A(t)=0[4]$.

Considering the expression above of the Jacobi operator $\bar{R}_{\gamma}$, one can solve explicitely the Jacobi equation, and obtain the endomorphism-valued function $\bar{A}$ to be a block diagonal matrix of the form

$$
\overline{A(t)}=\left(\begin{array}{cccc}
P(t) & & & \\
& P(t) & & \\
& & P(t) & \\
& & & t I_{4 n-8}
\end{array}\right),
$$

with $P(t)$ the $2 \times 2$ matrix

$$
P(t)=\left(\begin{array}{cc}
-\frac{c}{8} g(X, X) t^{3}+t & \frac{c}{8} g(X, X) t^{3} \\
-\frac{c}{8} g(X, X) t^{3} & \frac{c}{8} g(X, X) t^{3}+t
\end{array}\right) .
$$

Hence $\operatorname{det} \overline{A(t)}=t^{4 n-2}$, which shows the nonexistence of conjugate points along $\gamma$.

REMARK 13. If $\gamma$ is a nonnull geodesic, and the $q$-sectional curvature is constant $c$, one can also show the nonexistence of conjugate points along geodesics $\gamma$ with $c\left\langle\gamma^{\prime}, \gamma^{\prime}\right\rangle<0$, and the existence of a conjugate point of zero at each $t=\left(c\left\langle\gamma^{\prime}, \gamma^{\prime}\right\rangle\right)^{-1 / 2} k \pi$, for positive values of $k$, if $c\left\langle\gamma^{\prime}, \gamma^{\prime}\right\rangle>0$.

\section{References}

[1] Barros, M. and Romero, A., Indefinite Kähler manifolds, Math. Ann., 261 (1982), 55-62.

[2] Besse, A., Einstein manifolds, Erg. G., Folge 10, Springer-Verlag, Berlin, Heidelberg, New York, 1987.

[3] Bonome, A., Castro, R., García-Río, E. and Hervella, L. M., On the holomorphic sectional curvature of an indefinite Kähler manifold, C.R. Acad. Sci. Paris, 315 (1992), 1183-1187.

[4] Kupeli, D. N., On conjugate and Focal Points in Semi-Riemannian Geometry, Math., Z., 198 (1988), 569-589. 
5] Nomizu, K., Remarks on sectional curvature of an indefinite metric, Proc. Amer. Math. Soc., 89 (1983), 473-476.

[6] Pérez, J.D. and Santos, F., Indefinite quaternion space forms, Ann. Mat. Pura Appl., 132 (1982), 383-398.

[7] Yano, K. and Kon, M., Structures on Manifolds, Series in Pure Mathematics 3, World Scientific Publ. Co., Singapore, 1984.

Authors addresses

E. García-Río

Departamento de Análise Matemática

Facultade de Matemáticas

Universidade de Santiago de Compostela

15706 Santiago (SPAIN)

E-mail : eduardo@zmat.usc.es

M. E. Vázquez-Abal

Departamento de Xeometría e Topoloxía

Facultade de Matemátices

Universidade de Santiago de Compostela

15706 Santiago (SPAIN)

E-mail : meva@zmat.usc.es 